\title{
Protective effect of hydrogen gas inhalation on radiation-induced bone marrow damage in cancer patients: a retrospective observational study
}

\section{Shin-ichi Hirano}

Miz Company Limited

\section{Yukimasa Aoki}

Clinic C4

\section{Ryosuke Kurokawa}

Miz Company Limited

\section{Xiao-Kang Li}

National Institute for Child Health and Development

Naotsugu Ichimaru

Osaka University graduate school of medicine

\section{Shiro Takahara}

Kansai Medical Hospital

Yoshiyasu Takefuji ( $\nabla$ takefuji@keio.jp )

Keio University https://orcid.org/0000-0002-1826-742X

\section{Research}

Keywords: Intensity-modulated radiation therapy, Hydrogen gas, Bone marrow damage, Cancer patient, Retrospective observational study

Posted Date: March 9th, 2020

DOI: https://doi.org/10.21203/rs.3.rs-16275/v1

License: (a) (1) This work is licensed under a Creative Commons Attribution 4.0 International License. Read Full License 


\section{Abstract}

Background: Although intensity-modulated radiation therapy (IMRT) has been developed as an alternative to conventional radiotherapy, but reducing bone marrow damage is limited in the patients with multiple tumor lesions and large irradiation volume. Thus, novel technology is needed to mitigate further IMRTinduced bone marrow damage. Molecular hydrogen $(\mathrm{H} 2)$ was recently reported as a preventive and therapeutic antioxidant that selectively scavenges hydroxyl radical $(\cdot \mathrm{OH})$ and peroxynitrite (ONOO-). This observational study aims to examine whether $\mathrm{H} 2$ gas treatment improves IMRT-induced bone marrow damage in cancer patients.

Methods: The patients with end stage of cancer were received IMRT once per day for 1 to 4 weeks except Saturday and Sunday. After each course of IMRT, the patients of control group $(n=7)$ were housed in health care chamber ( $\mathrm{HCC}$, mild hyperbaric oxygen chamber) for 30 minutes, and the patients of $\mathrm{H} 2$ group $(n=16)$ were also housed in HCC and received $5 \% \mathrm{H} 2$ gas for 30 minutes once per day. Radiationinduced bone marrow damage was evaluated by hematological examination of peripheral blood obtained before and after IMRT, and the data was expressed the ratio of after treatment to before treatment.

Results: The total number of radiation courses and total exposure doses of radiation were similar between the control and $\mathrm{H} 2$ groups. IMRT with HCC therapy significantly reduced white blood cells (WBC) and platelets (PLT), but not red blood cells (RBC), hemoglobin (HGB) and hematocrit (HT). In contrast, $\mathrm{H} 2$ gas treatment significantly alleviates reducing effects of WBC and PLT $(p=0.0011$ and $p=0.0275$, respectively). Bone marrow damage index calculated by total exposure dose and WBC ratio also demonstrated the radioprotective effects of $\mathrm{H} 2$ gas $(p=0.0231)$. Tumor responses to IMRT were similar between the two groups, and 4 of 7 (57\%) patients in the control group and 7 of $16(44 \%)$ patients in the $\mathrm{H} 2$ group achieved a complete response (CR) or partial response (PR). In addition, 3 of $16(19 \%)$ patients in the $\mathrm{H} 2$ group achieved stable disease (SD).

Conclusion: The results obtained demonstrated that $\mathrm{H} 2$ gas inhalation therapy alleviated IMRT-induced bone marrow damage without compromising the anti-tumor effects of IMRT. The present study suggests that this novel approach of $\mathrm{H} 2$ gas inhalation therapy may be applicable to IMRT-induced bone marrow damage in cancer patients.

\section{Introduction}

When the shape of a tumor is irregular and complex, conventional radiotherapy is unable to maintain a high tumor control rate and simultaneously attenuate the associated complications, because normal tissues and organs surrounding the tumor receive the same dose of irradiation. As an alternative to conventional radiotherapy, intensity-modulated radiation therapy (IMRT) has been developed to only irradiate tumors [1]. However, bone marrow damage still occurs in cancer patients with multiple tumor lesions and large irradiation volumes. Thus, novel technology is needed to mitigate further IMRT-induced bone marrow damage. To attenuate the symptoms associated with end-stage cancer, patients are also 
housed in a health care chamber (HCC, a mild hyperbaric oxygen chamber) [2]; however, satisfactory outcomes have not yet been achieved.

The direct effects of radiation are due to the changes it induces in bioactive macromolecules such as proteins and nucleic acids. Ionization, excitation, chemical bond rupture, and changes in molecular structures occur during this process, leading to abnormal functions and metabolic disorders [3]. Indirect effects also occur via the generation of free radicals by water radiolysis. Hydroxyl radicals $(\cdot \mathrm{OH})$, the strongest reactive oxidant species (ROS), are formed from this radiolysis and react with nucleic acids, lipids, and proteins. Approximately $65 \%$ of DNA damage is caused by the indirect effects of free radicals such as $\cdot \mathrm{OH}[4]$. Therefore, selective and high concentrations of $\cdot \mathrm{OH}$ scavengers have potential as radioprotective agents.

Molecular hydrogen $\left(\mathrm{H}_{2}\right)$ was recently identified by Ohsawa et al. as a preventive and therapeutic antioxidant that selectively scavenges $\cdot \mathrm{OH}$ and peroxynitrite $\left(\mathrm{ONOO}^{-}\right)$[5]. However, in 2005, 2 years before Ohsawa's study, Yanagihara et al. reported that the consumption of neutral $\mathrm{H}_{2}$-rich water produced by electrolysis may effectively reduce the oxidative stress induced by chemical oxidants in rats, which is pioneering research in $\mathrm{H}_{2}$ medicine [6]. Due to its small size and electrically neutral properties, $\mathrm{H}_{2}$ easily reaches target organs. $\mathrm{H}_{2}$ has also been proposed as a treatment for various oxidative stress-related diseases [7-9]. Previous studies demonstrated that $\mathrm{H}_{2}$ exerted radioprotective effects in various animal models $[10,11]$ and improved the quality of life $(\mathrm{QOL})$ of patients treated with radiotherapy for liver tumors [12]. However, there is currently no definitive therapy to attenuate radiation-induced bone marrow damage in cancer patients. Therefore, this retrospective observational study investigated whether $\mathrm{H}_{2}$ gas treatment mitigates IMRT-induced bone marrow damage in cancer patients.

\section{Methods}

\section{Subjects and designs}

The study was a retrospective observational examination performed at Clinic C4 in Tokyo, Japan between May 2015 and November 2016. During this period, 26 patients with end-stage cancer received two different treatments, IMRT with or without $\mathrm{H}_{2}$ gas inhalation (the $\mathrm{H}_{2}$ group and control group, respectively). Data were collected from 23 patients because one patient in the control group and two patients in the $\mathrm{H}_{2}$ group were excluded based on the selection criteria of the present study.

Patients were subjected to 5 to 20 minutes of Tomo Therapy (Japan Accuray Inc., Tokyo, Japan) once per day for 1 to 4 weeks, except Saturday and Sunday. After each course of IMRT, the control group $(n=7,3$ men and 4 women) was housed in HCC (APF2, Air Press Co., Ltd., Tokyo, Japan) for 30 minutes under the environmental conditions of 1.35 atm and $27 \% \mathrm{O}_{2}$. The $\mathrm{H}_{2}$ group $(n=16,8$ men and 8 women) were also housed in $\mathrm{HCC}$ and received $5 \% \mathrm{H}_{2}$ gas inhalation for 30 minutes. $\mathrm{H}_{2}$ gas was prepared by mixing $\mathrm{H}_{2}$ gas and air using a 3.5\% $\mathrm{H}_{2}$ gas inhaler (MHG-2000, MiZ Co., Ltd., Kanagawa, Japan) and $6.5 \% \mathrm{H}_{2}$ gas inhaler 
(MHG-2000a, MiZ Co., Ltd.) connected in parallel. Both $\mathrm{H}_{2}$ gases were produced by the electrolysis of water, and concentrations were controlled under the detonation limit of the mixture of $\mathrm{H}_{2}$ gas and air [13]. Patients received mixed gas via a nose cannula at a flow rate of $4 \mathrm{~L} /$ minute in $\mathrm{HCC}$.

\section{Patient Characteristics}

Patient characteristics, including sex, age, tumor origin, stage of cancer, and number of metastases, are shown in Table 1 and additional file 1 (Table S1), and were not significantly different between the control and $\mathrm{H}_{2}$ groups.

Insert Table 1 here.

As shown in Fig. 1a and $1 \mathrm{~b}$, the number of radiation courses and total exposure doses of radiation $\left(\times 10^{3}\right.$ $\mathrm{cm}^{3}$. Gy) for the control and $\mathrm{H}_{2}$ groups were $10.7 \pm 1.3$ and $11.2 \pm 1.1$, and $446.0 \pm 101.8$ and $410.2 \pm$ 124.4 , respectively, which were not significantly different between the two groups $(p=0.8064$ and $p=$ 0.8607 , respectively).

\section{Evaluation Of Bone Marrow Damage}

Bone marrow damage was evaluated by a hematological examination of peripheral blood. Blood samples were obtained from all patients on the first day of IMRT (before the treatment) and after 1 to 4 weeks of IMRT (after the treatment). A blood hematology test for the counts of red blood cells (RBC), white blood cells (WBC), and platelets (PLT), the concentration of hemoglobin (HGB), and the hematocrit $(\mathrm{HT})$ was conducted before and after the treatment using standard assays in a contract clinical examination laboratory.

\section{Evaluation Of Tumor Responses}

Complete response (CR) was defined as the disappearance of any intratumoral arterial enhancement in all target lesions. Partial response (PR) was defined as at least a $30 \%$ decrease in the sum of the diameters of viable target lesions. Progressive disease (PD) was defined as at least a $20 \%$ increase in the sum of the diameters of viable target lesions or the appearance of a new lesion. Stable disease (SD) was defined as a tumor status that did not meet any of the above criteria.

\section{Statistical analysis}

Hematological data were expressed as ratios of after to before the treatment. We also analyzed the relationship between the total exposure dose and bone marrow damage. This relationship was calculated as $A / B$ or $A / C$, where $A$ is the total radiation dose and $B$ or $C$ is the WBC ratio or PLT ratio, respectively. These data were presented as means \pm SEM (means with standard errors) and analyzed statistically by 
an unpaired Student's t-test or the Mann-Whitney U-test where appropriate using BellCurve for Excel (Version 3·0, Social Survey Research Information Co., Ltd., Japan). P < 0.05 was considered to be significant.

\section{Results}

\section{Tumor responses}

Tumor responses to IMRT were similar between the two groups, and 4 of 7 (57\%) patients in the control group and 7 of 16 (44\%) patients in the $\mathrm{H}_{2}$ group achieved a CR or PR. In addition, 3 of 16 (19\%) patients in the $\mathrm{H}_{2}$ group achieved SD. These results indicated that tumor responses to IMRT were similar between the two groups, and that $\mathrm{H}_{2}$ gas inhalation did not compromise the anti-tumor effects of IMRT. Moreover, the QOL, such as fatigue, sleep, and gastrointestinal symptoms, was similar between the two groups, indicating that $\mathrm{H}_{2}$ gas inhalation did not compromise the QOL of patients receiving IMRT.

\section{Bone Marrow Damage}

Bone marrow damage was evaluated by a hematological examination of peripheral blood. Blood samples were obtained from all patients on the first day of IMRT (before treatment) and after 1 to 4 weeks of IMRT (after treatment). In the control and $\mathrm{H}_{2}$ treatment groups, $\mathrm{RBC}$ ratios were $1.108 \pm 0.124$ and $0.985 \pm 0.027$, HGB ratios were $1.078 \pm 0.126$ and $0.966 \pm 0.029$, and HT ratios were $1.073 \pm 0.121$ and $0.953 \pm 0.027$, respectively. These values were close to 1 , and no significant differences were observed between the two groups (Fig. 2a, 2b, and 2c), indicating that IMRT did not influence these hematological markers. WBC ratios were $0.145 \pm 0.041$ and $0.612 \pm 0.088$ for the control and $\mathrm{H}_{2}$ groups, respectively, and were significantly different $(p=0.0011$; Fig. $2 d)$. PLT ratios were $0.337 \pm 0.106$ and $0.752 \pm 0.130$ for control and $H_{2}$ groups, respectively, and were also significantly different $(p=0.0275$; Fig. 2e). These results indicated that IMRT selectively reduced hematological markers, such as WBC and $\mathrm{PLT}$, in the control group, whereas the $\mathrm{H}_{2}$ gas treatment protected against these reductions.

The WBC damage index (the relationship between the total exposure dose and WBC ratio) for control and $\mathrm{H}_{2}$ groups were $7012 \pm 3244$ and $1309 \pm 682$, respectively, and were significantly different $(p=0.0231$; Fig. 3a). The PLT damage index (the relationship between the total exposure dose and PLT ratio) for control and $\mathrm{H}_{2}$ groups were $7008 \pm 4827$ and $956 \pm 352$, respectively, and were not significantly different $(\mathrm{p}=0.1606$; Fig. $3 b)$. These results indicated that IMRT increased the WBC damage index, whereas the $\mathrm{H}_{2}$ gas treatment prevented this increase.

\section{Discussion}

IMRT has been performed for cancer patients to alleviate the adverse effects associated with increased oxidative stress and inflammation [1]; however, bone marrow damage still occurs in patients with multiple 
tumor lesions and large irradiation volumes. The present study investigated whether $\mathrm{H}_{2}$, a selective $\cdot \mathrm{OH}$ scavenger, mitigates IMRT-induced bone marrow damage in end-stage cancer patients. The results obtained demonstrated that the $\mathrm{H}_{2}$ gas treatment alleviated IMRT-induced bone marrow damage without compromising the anti-tumor effects of IMRT. To the best of our knowledge, this is the first study to report the benefits of $\mathrm{H}_{2}$ gas on IMRT in cancer patients.

Since the number of radiation courses and total exposure doses of radiation need to be selected according to the size and number of tumors in individual patients, the number of radiation courses performed in the present study ranged between a minimum of 5 ( 1 week) and maximum of 20 (4 weeks). Therefore, blood sampling to examine the adverse effects of radiation was performed from 1 to 4 weeks. However, the average number of radiation courses and the average total exposure doses of radiation ( $x$ $10^{3} \mathrm{~cm}^{3} \cdot \mathrm{Gy}$ ) in the control and $\mathrm{H}_{2}$ groups were equivalent, indicating that the design of the present study allowed for the protective effects of $\mathrm{H}_{2}$ gas inhalation to be evaluated.

The present study demonstrated that the QOL, such as fatigue, depression, sleep, and gastrointestinal symptoms, was similar between the control and $\mathrm{H}_{2}$ groups. These results indicate that the inhalation of $\mathrm{H}_{2}$ gas did not improve the QOL. However, since the main purpose of the present study was to mitigate radiation-induced damage, long-term inhalation may be needed to improve the QOL. Moreover, although the effects of $\mathrm{H}_{2}$ gas inhalation were not examined in a non-radiated control group (patients not undergoing radiotherapy), based on the findings of an animal study showing that $\mathrm{H}_{2}$-rich saline did not affect hematological data in non-radiated control mice [11], $\mathrm{H}_{2}$ gas inhalation may not have an impact on hematological data in patients.

The radioprotective effects of $\mathrm{H}_{2}$ have been reported in different systems, including a cell-free system and various organs. In the cell-free system, Chuai et al. showed that the levels of $\cdot \mathrm{OH}$ produced by water radiolysis and the Fenton reaction were reduced by $\mathrm{H}_{2}$ solution [10]. Moreover, Yang et al. demonstrated that a $\mathrm{H}_{2}$-rich medium pretreatment decreased $\cdot \mathrm{OH}$ levels in $\mathrm{AHH}-1$ cells, a human lymphocyte cell line [11]. On the other hand, Yang et al. also noted radiation-induced hematological changes in WBC and PLT, but not in $\mathrm{RBC}, \mathrm{HGB}$, or the mean corpuscular volume (MCV) in mice subjected to total body radiation, and found that an intraperitoneal injection of $\mathrm{H}_{2}$-rich saline significantly attenuated the depletion of WBC and PLT [11]. They also showed that $\mathrm{H}_{2}$ reduced radiation-induced apoptosis in thymocytes and splenocytes in mice. These findings suggest that $\mathrm{H}_{2}$ reduced radiation-induced $\cdot \mathrm{OH}$ levels by directly affecting $\cdot \mathrm{OH}$ levels, and simultaneously reduced radiation-induced oxidative stress, apoptosis, and inflammation by indirect effects on $\cdot \mathrm{OH}$. In the present study, the $\mathrm{H}_{2}$ gas treatment in combination with HCC therapy did not suppress the anti-tumor effects of IMRT in cancer patients because the response rates between the two groups were similar. This result is supported by the findings of Kang et al., who demonstrated that the consumption of $\mathrm{H}_{2}$-rich water reduced radiation-induced oxidative stress and the QOL in patients treated with radiotherapy for liver tumors without compromising anti-tumor effects [12]. Therefore, the mechanisms underlying the radioprotective effects of $\mathrm{H}_{2}$ gas may involve not only direct 
effects on $\cdot \mathrm{OH}$, but also indirect effects on $\mathrm{OH}$ via the activation of the host-mediated antioxidant and anti-inflammation systems.

In the present study, patients received the $\mathrm{H}_{2}$ gas treatment after each course of IMRT. Previous studies reported the preventive $\left(\mathrm{H}_{2}\right.$ gas inhalation before radiation), but not therapeutic effects of this treatment. Furthermore, $\mathrm{H}_{2}$ selectively scavenges $\cdot \mathrm{OH}$ and $\mathrm{ONOO}^{-}$[5]; however, since these ROS are rapidly generated, many chemical agents generally exert stronger effects via prophylactic rather than therapeutic administration. Thus, the therapeutic administration protocol employed in the present study appears to accurately reflect the effects of $\mathrm{H}_{2}$ gas inhalation because the mechanisms underlying the radioprotective effects of $\mathrm{H}_{2}$ gas may involve not only direct, but also indirect effects on $\cdot \mathrm{OH}$ via the activation of host defense systems.

Hyperbaric oxygen therapy that involves housing patients for 1 hour in a chamber containing $100 \% \mathrm{O}_{2}$ at 2 atm has been performed, and is effective for patients with decompression, peripheral circulation failure, wound dysfunction, and radiation-induced damage. In contrast, therapy using HCC is based on the principle of hyperbaric oxygen therapy, and patients are housed in a chamber containing $1.35 \mathrm{~atm}$ and air [2]. In the present study, based on Henry's law, patients in the $\mathrm{H}_{2}$ group received 1.35-fold more $\mathrm{H}_{2}$ and $\mathrm{O}_{2}$ under 1.35 atm environmental conditions, which is equivalent to patients receiving $6.8 \% \mathrm{H}_{2}$ gas inhalation therapy under normal pressure. In additional experiments, the radioprotective effects of $6.5 \%$ $\mathrm{H}_{2}$ gas inhalation therapy at normal pressure (data not shown) were found to be similar, suggesting the importance of inhaling higher $\mathrm{H}_{2}$ concentrations to attenuate bone marrow damage. Although $\mathrm{H}_{2}$ gas concentrations for the detonation limit in a mixture of $\mathrm{H}_{2}$ and air are less than $4 \%$, we recently demonstrated that the detonation limit was less than $10 \%$ in our experiment [14] and a literature search [15]. Therefore, $6.5 \% \mathrm{H}_{2}$ gas therapy without $\mathrm{HCC}$ appears to be a clinically convenient, effective, and safe method for mitigating IMRT-induced bone marrow damage.

The development of safe and more effective radioprotective agents is very important in view of their potential application during radiotherapy for cancer patients. The radioprotective effects of many synthetic and natural agents have been investigated in the past 50 years. Nutraceuticals, including vitamin $\mathrm{C}$, vitamin E succinate, a-lipoic acid, and $\mathrm{N}$-acetyl cystine, in addition to hematopoietic growth factors and cytokines, such as stem cell factor, granulocyte colony-stimulating factor, granulocytemacrophage colony-stimulating factor, and interleukin 3, have been reported to exert radioprotective effects in animal models [16-20]. Amifostine, known as Ethyol or WR2721, is the only clinically accepted radioprotective agent, but is not considered to be a viable option as a radioprotective agent because of its inherent dose-limiting toxicities [21-23]. Although several drugs are in different stages of evaluation, none possess all of the requisite qualities of an optimum radioprotective agent. Thus, there are no safe and effective non-toxic radioprotective agents available for human use. Previous studies reported that $\mathrm{H}_{2}$ exerts radioprotective effects in various animal models $[10,11]$. Moreover, $\mathrm{H}_{2}$ improved the QOL of patients treated with radiotherapy for liver tumors [12]. However, there is currently no definitive therapy to improve radiation-induced bone marrow damage in cancer patients. Therefore, the present study 
investigated whether $\mathrm{H}_{2}$ gas mitigates IMRT-induced bone marrow damage in cancer patients, and the results obtained demonstrated that $\mathrm{H}_{2}$ gas inhalation therapy with or without HCC alleviated IMRTinduced bone marrow damage without compromising the anti-tumor effects of IMRT. Clinical studies have demonstrated that $\mathrm{H}_{2}$ has no adverse effects [7-9]. Therefore, these findings may provide the foundation for a clinically applicable, effective, and safe strategy for a $\mathrm{H}_{2}$ gas to mitigate IMRT-induced bone marrow damage.

Bone marrow damage, such as reductions in WBC (leukopenia) and PLT (thrombocytopenia), frequently occurs during cancer radiotherapy, including IMRT, and this is a limiting factor for radiotherapy [24, 25]. Since leukopenia and thrombocytopenia may cause infection and gastrointestinal bleeding, caution is required. If bone marrow damage may be attenuated by $\mathrm{H}_{2}$ gas inhalation, it will lead to the prevention of infection and gastrointestinal bleeding. Therefore, $\mathrm{H}_{2}$ gas inhalation may improve the prognosis of cancer patients.

\section{Conclusions}

In conclusion, the present study investigated whether $\mathrm{H}_{2}$ gas inhalation mitigates IMRT-induced bone marrow damage in cancer patients. The results obtained demonstrated that $\mathrm{H}_{2}$ gas inhalation therapy alleviated IMRT-induced bone marrow damage without compromising the anti-tumor effects of IMRT. However, this study had some limitations related to the number of patients, the retrospective observational analysis, and data collection from a single hospital. Although further large-scale clinical studies involving many hospitals are required, the present study suggests that this novel approach of $\mathrm{H}_{2}$ gas inhalation therapy may be applicable to IMRT-induced bone marrow damage in cancer patients.

\section{Abbreviations}

IMRT: intensity-modulated radiation therapy; $\mathrm{H}_{2}$ : hydrogen; WBC: white blood cell; $\mathrm{RBC}$ : red blood cell; HGB: hemoglobin; PLT: platelet; HT: hematocrit; HCC: health care chamber; OH: hydroxyl radical; ROS: reactive oxidant species; $\mathrm{ONOO}^{-}$: peroxynitrite; $\mathrm{QOL}$ : quality of life; $\mathrm{CR}$ : complete response; PR: partial response; SD: stable disease; PD: progressive disease; MCV: mean corpuscular volume; SEM: means with standard errors; M: male; F; female; meta: metastasis; disse: dissemination; NS: not significant.

\section{Declarations}

\section{Acknowledgments}

The authors are grateful to Mr. Fumitake Sato, Ms. Yoko Sato, Dr. Yusuke Ichikawa, and Mr. Masatsugu Saito (MiZ Co., Ltd.) for their excellent advice on the writing of this manuscript.

\section{Authors' contributions}


$\mathrm{SH}$ and YA designed the study and analyzed the data. SH wrote the main text, analyzed the data, and prepared figures. RK developed and prepared the $\mathrm{H}_{2}$ gas inhaler. $\mathrm{YT}, \mathrm{YA}, \mathrm{X}-\mathrm{KL}, \mathrm{NI}$, and ST supported this study by collecting data and giving advice. All authors read and approved the final manuscript.

\section{Funding}

Self-funding.

\section{Availability of data materials}

All data included in this study are available upon request by contact with the corresponding author.

\section{Ethics approval and content to participate}

The study protocol and materials were approved by an Ethics Committee review of Tokyo Clinic and Research Institute ICVS Incorporated (Tokyo, Japan), and all patients provided written informed consent prior to receiving therapy. All methods were performed in accordance with the relevant guidelines and regulations. The present study was registered in the University Hospital Medical Information Network (UMIN) Clinical Trials Registry (UMIN ID: UMIN000035864) on February 20, 2019.

\section{Consent for publication}

Not applicable.

\section{Competing interests}

The authors declare that they have no competing interests.

\section{Author details}

${ }^{1}$ MiZ Company Limited, 2-19-15 Ofuna, Kamakura, Kanagawa 247-0056, Japan. ${ }^{2}$ Clinic C4, 33-12 Motoyoyogicho, Shibuya-ku, Tokyo 151-0062, Japan. ${ }^{3}$ Division of Transplantation Immunology, National Institute for Child Health and Development, 2-10-1 Okura, Setagaya, Tokyo 157-8535, Japan. ${ }^{4}$ Department of Advanced Technology for Transplantation, Osaka University Graduate School of Medicine, 2-2 Yamadaoka, Suita, Osaka 565-0871, Japan. ${ }^{5}$ Department of Renal Transplantation Center, Kansai Medical Hospital, 1-1-7-2 Shinsenrinishimachi, Toyonaka-city, Osaka 560-0083, Japan. ${ }^{6}$ Faculty of Environment and Information Studies, Keio University, 5322 Endo, Fujisawa 252-0882, Japan.

\section{References}

1. Fischer-Valuck BW, Rao YJ and Michalski JM. Intensity-modulated radiotherapy for prostate cancer. Transl Androl Uro. 2018; 7 (3): 297-307. 
2. Sidik S, Hardjodisastro D, Setiabudy R and Gondowiardjo S. Does hyperbaric oxygen administration decrease side effect and improve quality of life after pelvic radiation? Acta Med Indones. 2007; 39 (4): 169-73.

3. Shao L, Luo Y and Zhou D. Hematopoietic stem cell injury induced by ionizing radiation. Antioxid Redox Signal. 2014; 20 (2): 1447-62.

4. Ward JF. DNA damage produced by ionizing radiation in mammalian cells: identities, mechanisms of formation, and reparability. Prog Nucleic Acid Res Mol Biol. 1988; 35: 95-125.

5. Ohsawa I, Ishikawa M, Takahashi K, Watanabe M, Nishimaki K, Yamagata K, et al. Hydrogen acts as a therapeutic antioxidant by selectively reducing cytotoxic oxygen radicals. Nat Med. 2007; 13 (6): 688-94.

6. Yanagihara T, Arai K, Miyamae K, Sato B, Shudo T, Yamada M, et al. Electrolyzed hydrogen-saturated water for drinking use elicits an antioxidative effect: a feeding test with rats. Biosci Biotechnol Biochem. 2005; 69 (10): 1985-87.

7. Yoritaka A, Takanashi M, Hirayama M, Nakahara T, Ohta S, Hattori N. Pilot study of $\mathrm{H} 2$ therapy in Parkinson's disease. A randomized double-blind placebo-controlled trial. Mov Disord. 2013; 28 (6): 836-9.

8. Nishimaki K, Asada T, Ohsawa I, Nakajima E, Ikejima C, Yokota T, et al. Effects of molecular hydrogen assessed by an animal model and a randomized clinical study on mild cognitive impairment. Alzheimer Res. 2018; 15 (5): 482-92.

9. Ishibashi T, Sato B, Rikitake M, Seo T, Kurokawa R, Hara Y, et al. Consumption of water containing a high concentration of molecular hydrogen reduces oxidative stress and disease activity in patients with rheumatoid arthritis: an open-label pilot study. Med Gas Res. 2012; 2 (1):

10. Chuai Y, Gao F, Li B, Zhao L, Qian L, Cao F, et al. Hydrogen-rich saline attenuates radiation-induced male germ cell loss in mice through reducing hydroxyl radicals. Biochem J. 2012; 442 (1): 49-56.

11. Yang Y, Li B, Liu C, Chuai Y, Lei J, Gao F, et al. Hydrogen-rich saline protects immunocytes from radiation-induced apoptosis. Med Sci Monit. 2012; 18 (4): BR144-8.

12. Kang KM, Kang YN, Choi IB, Gu Y, Kawamura T. Toyoda Y, et al. Effects of drinking hydrogen-rich water on the quality of life of patients treated with radiotherapy for liver tumors. Med Gas Res. 2011; 1 (1): 11.

13. Kurokawa R, Seo T, Sato B, Hirano S, Sato F. Convenient methods for ingestion of molecular hydrogen: drinking, injection, and inhalation. Med Gas Res. 2015; 5:13.

14. Kurokawa R, Hirano S, Ichikawa Y, Matsuo G, Takefuji Y. Preventing explosions of hydrogen gas inhalers. Med Gas Res. 2019; 9 (3): 160-62.

15. Thomas G.O. Flame acceleration and the development of detonation in fuel-oxygen mixtures at elevated temperatures and pressures. J Hazard Mat. 2009; 163 (2-3): 783-94.

16. Sato T, Kinoshita M, Yamamoto T, Ito M, Nishida T, Takeuchi M, et al, Treatment of irradiated mice with high-dose ascorbic acid reduced lethality. PLoS ONE. 2015; 10 (2): e0117020. 
17. Drouet M, Mourcin F, Grenier N, Leroux V, Denis J, Mayol JF, et al. Single administration of stem cell factor, FLT-3 ligand, megakaryocyte growth and development factor, and ineterleukin-3 in combination soon after irradiation prevents nonhuman primates from myelosuppression: long-term follow-up of hematopoiesis. Blood. 2004; 103 (3): 878-85.

18. Farese AM, Casey DB, Smith WG, Vigneulle RM, McKearn JP, MacVittie TJ, et al. Leridistim, a chimeric dual G-CSF and IL-3 receptor agonist, enhances multilineage hematopoietic recovery in a nonhuman primate model of radiation-induced myelosuppression: effect of schedule, dose, and route of administration. Stem cell. 2001; 19 (6): 522-33.

19. Herodin F, Bourin P, Mayol JF, Lataillade JJ, Drouet M, Short-term injection of antiapoptotic cytokine combinations soon after lethal gamma-irradiation promotes survival. Blood. 2003; 101 (7): 2609-16.

20. MacVittie TJ, Farese AM, Smith WG, Baum CM, Burton E, McKearn JP. Myelopoietin, an engineered chimeric IL-3 and G-CSF receptor agonist, stimulates multilineage hematopoietic recovery in a nonhuman primate model of radiation-induced myelosuppression. Blood. 2000; 95 (3): 837-45.

21. Seed TM, Fry SA, Neta R, Weiss JW, Jarrett DG, Thomassen D. Prevention and treatments: Summary statement. Milit Med. 2002; 167 (2 Suppl): 87-93.

22. Thorstad WI, Haughey B, Chao KS-C. Pilot study of subcutaneous amifostine in patients undergoing postoperative intensity modulated radiation therapy for head and neck cancer: preliminary data. Semin Oncol. 2003; 30 (6 Suppl 18): 96-100.

23. Seed TM, Inal CE, Singh VK, Radioprotection of hematopoietic progenitors by low dose amifostine prophylaxis. Int J Radiation Biol. 2014; 90 (7): 594-604.

24. Byun HK, Kim N, Yoon HI, Kan SG, Kim SH, Cho J, et al. Clinical predictors of radiation-induced lymphopenia in patients receiving chemoradiation for glioblastoma: clinical usefulness of intensitymodulated radiotherapy in the immune-oncology era. Radiat Oncol 2019; 14 (1): 51.

25. Ye L, Oei RW, Kong F, Xu T, Shen C, Wang X, et al. Prognostic values of hematological biomarkers in nasopharyngeal carcinoma patients treated with intensity-modulated radiotherapy. Eur Arch Otorhinolaryngol. 2018; 275 (5): 1309-17.

\section{Supplementary Files Legend}

Additional file 1. Table S1. Patient characteristics.

\section{Table}

Due to technical limitations, Table 1 is only available for download from the Supplementary Files section.

\section{Figures}


a

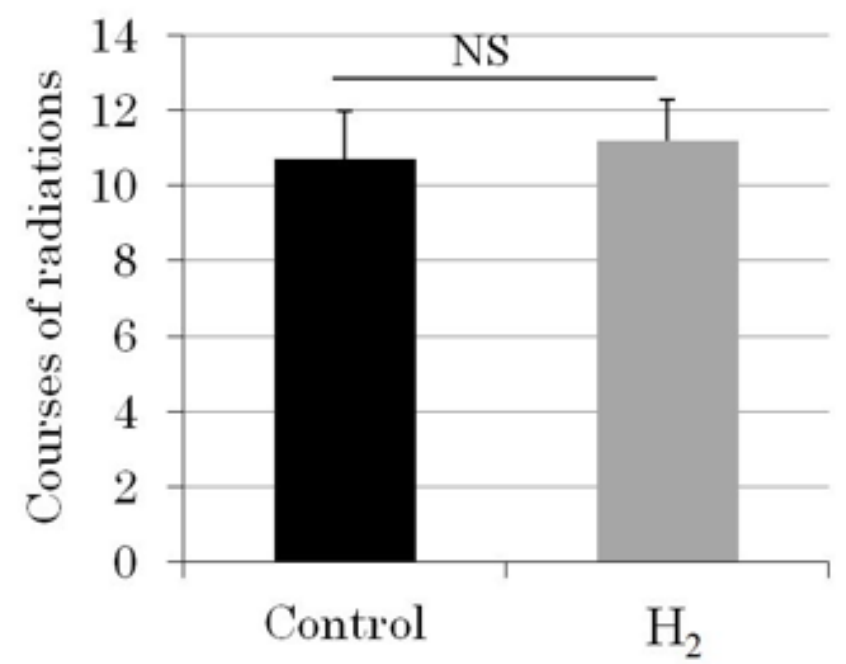

b

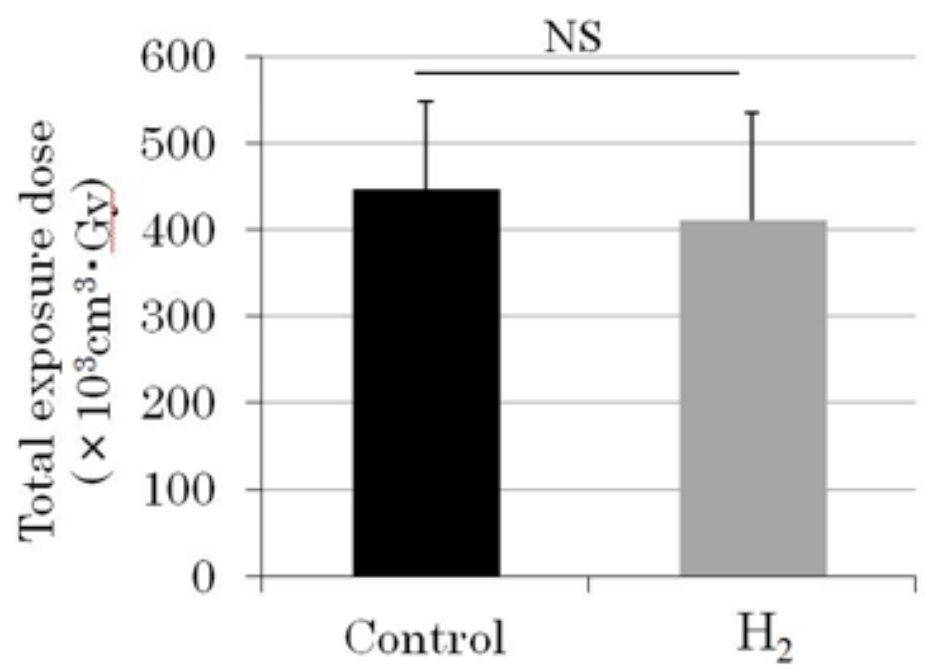

Figure 1

Radiation Conditions: Number of radiation courses for the control group and hydrogen ( $\mathrm{H} 2)$ group (a) and total exposure doses of radiation $(\times 103 \mathrm{~cm} 3 \cdot \mathrm{Gy})$ for these groups (b). NS: not significant. Data are given as means \pm SEM.

a

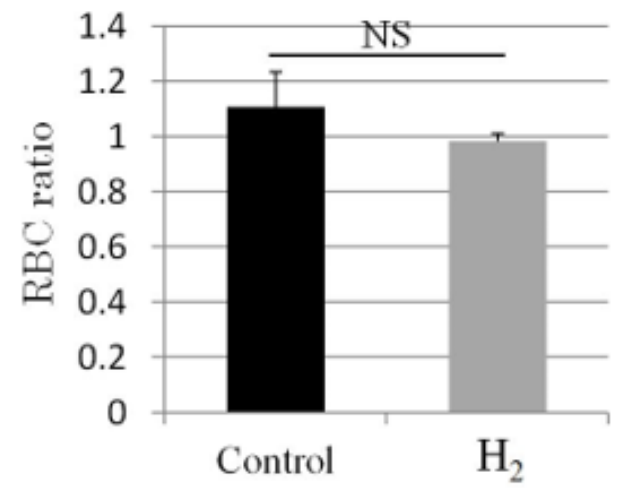

$\mathrm{b}$

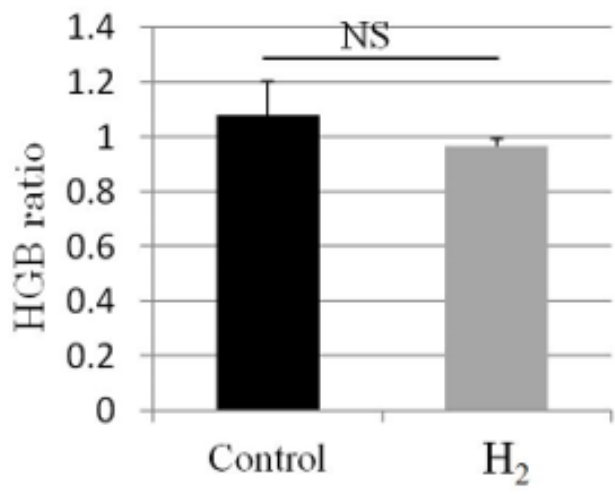

$\mathrm{C}$

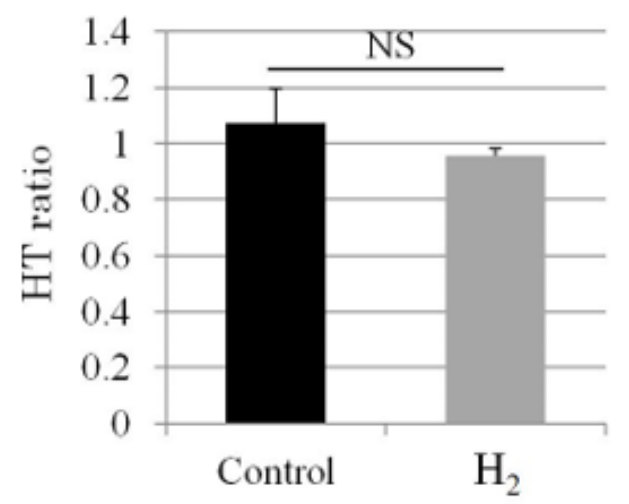

d

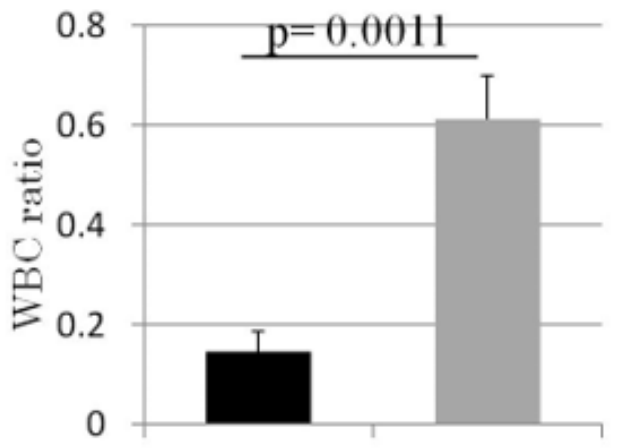

Control
$\mathrm{H}_{2}$ e

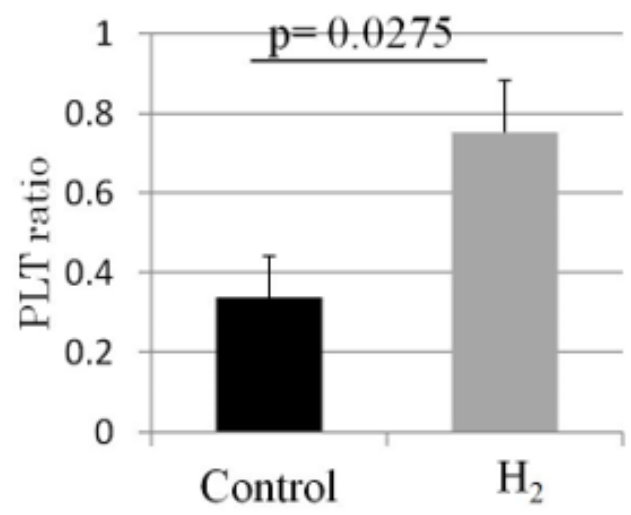


Figure 2

Effects on Hematological Data: Effects of the hyperbaric hydrogen $(\mathrm{H} 2)$ gas treatment on red blood cell counts (RBC), hemoglobin levels (HGB), the hematocrit (HT), white blood cell counts (WBC), and platelet counts (PLT). Blood samples were obtained from all patients on the first day of intensity-modulated radiation therapy (IMRT) (before treatment) and after 1 to 4 weeks of IMRT (after treatment). Blood hematology tests were conducted before and after the treatment. RBC (a), HGB (b), HT (c), WBC (d), and PLT (e) were expressed as ratios of after to before the treatment. NS: not significant. Relative ratios are given as means \pm SEM.

a

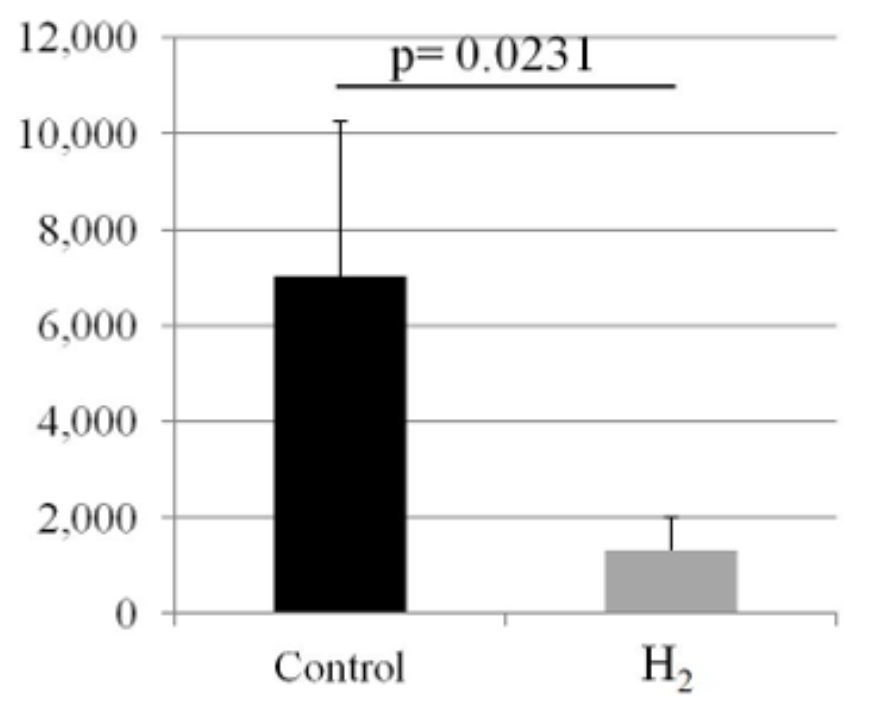

$\mathrm{b}$

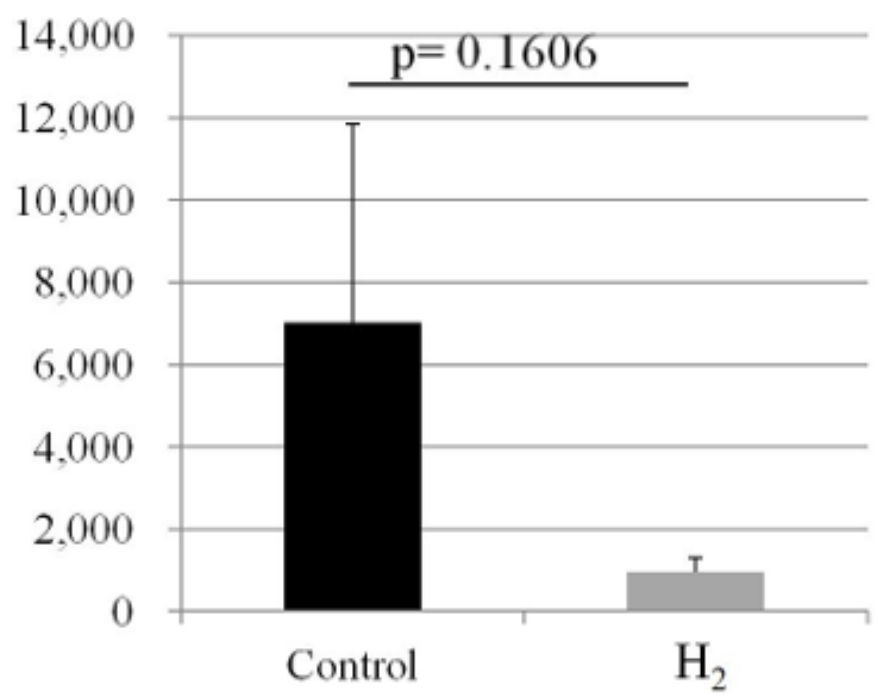

\section{Figure 3}

Effects on the bone marrow damage index: We analyzed the relationship between the total exposure dose and bone marrow damage, as shown in the Methods section. The relationship is calculated as A/B or $A / C$, where $A$ is the total radiation dose and $B$ or $C$ is the white blood cell (WBC) or platelet (PLT) ratio, respectively. The relationship between the total exposure dose and WBC ratio (WBC damage index) (a), and that between the total exposure dose and the PLT ratio (PLT damage index) (b) are shown. Data are given as means \pm SEM.

\section{Supplementary Files}

This is a list of supplementary files associated with this preprint. Click to download.

- supptable.pdf

- Table1.pdf 